\title{
Clinicopathological characteristics and outcomes in men with mesothelioma of the tunica vaginalis testis: analysis of published case-series data
}

\author{
Josias Bastian Grogg ${ }^{1}$. Jordi Nicola Fronzaroli ${ }^{1} \cdot$ Pedro Oliveira $^{2} \cdot$ Peter-Karl Bode ${ }^{1}$ Anja Lorch ${ }^{1}$. \\ Allaudin Issa ${ }^{2}$. Joerg Beyer ${ }^{3}$. Daniel Eberli ${ }^{1}$ - Vijay Sangar ${ }^{2} \cdot$ Thomas Hermanns $^{1} \cdot$ Noel William Clarke $^{2,4}$. \\ Christian Daniel Fankhauser ${ }^{1,2}$ (1)
}

Received: 22 November 2020 / Accepted: 12 January 2021 / Published online: 9 February 2021

(c) The Author(s) 2021

\begin{abstract}
Purpose Mesothelioma of the tunica vaginalis testis (MTVT) is a rare tumor, and currently, there are no published treatment recommendations.

Methods We performed a systematic literature review and synthesized clinical presentation, clinicopathological factors associated with metastatic disease, treatment options, and outcomes in men with MTVT.

Results We included 170 publications providing data on 275 patients. Metastatic disease occurred in 84/275 (31\%) men with malignant MTVT: Most common sites included retroperitoneal lymph nodes (LNs) (40/84, 48\%), lungs (30/84, 36\%), and inguinal LNs $(23 / 84,27 \%)$.

Invasion of the spermatic cord or scrotum was the only risk factor for local recurrence [odds ratio (OR) 3.21, 95\% confidence interval (CI) 1.36-7.57]. Metastatic disease was associated with age $\geq 42$ years (OR 3.02, 95\% CI 1.33-6.86), tumor size $\geq 49 \mathrm{~mm}$ (OR 6.17, 95\% CI 1.84-20.74), presence of necrosis (OR 8.31, 95\% CI 1.58-43.62), high mitotic index (OR $13.36,95 \%$ CI 1.53-116.51) or angiolymphatic invasion (OR 3.75, 95\% CI 1.02-13.80), and local recurrence (OR 4.35, 95\% CI 2.00-9.44). Complete remission in the metastatic setting was observed in five patients, most of whom were treated with multimodal therapy. Median survival in patients with metastatic disease was 18 months (IQR 7-43).

Conclusion Malignant MTVT is a rare but aggressive disease. Since local recurrence is a risk factor for metastatic progression, we recommend aggressive local treatment. Survival and response to any treatment in the metastatic setting are limited.
\end{abstract}

Keywords Urology $\cdot$ Testis cancer $\cdot$ Mesothelioma $\cdot$ Orchiectomy $\cdot$ Testis-sparing surgery $\cdot$ Systematic review

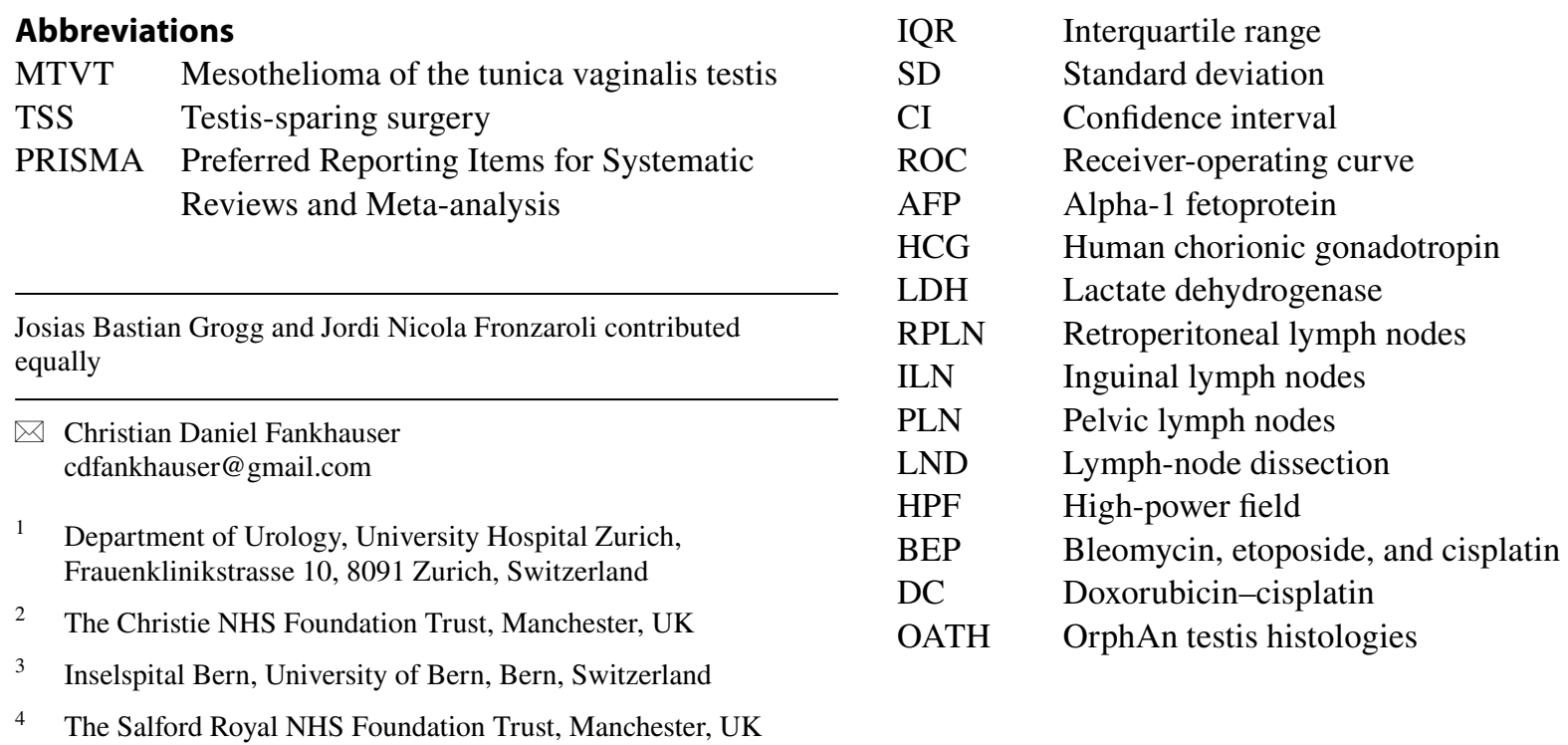




\section{Introduction}

Mesothelioma typically involves the pleura or peritoneum and only rarely the tunica vaginalis testis (Gurdal and Erol 2001; Plas et al. 1998a). Due to the low prevalence of mesothelioma of the tunica vaginalis testis (MTVT), treatment recommendations have not been developed. We collated published case reports of men with MTVT to describe clinicopathological factors associated with local recurrence or metastatic disease as well as outcome from treatments used.

\section{Methods}

\section{Evidence acquisition}

\section{Data acquisition and search strategy}

A systematic literature review was performed using the Preferred Reporting Items for Systematic Reviews and Meta-analysis (PRISMA) statement (Moher et al. 2009, 2015). Prior to data acquisition, the study protocol/search strategy was published on the PROSPERO registry (http:// www.crd.york.ac.uk/PROSPERO; Registration Number CRD42019129595).

Our search identified articles published up to 14th January 2019 and utilised the following electronic databases: MEDLINE $^{\circledR}$, Embase ${ }^{\circledR}$, Scopus $^{\circledR}$, the Cochrane Database of Systematic Reviews, and Web of Science. To identify all relevant articles, a clinical medical librarian applied a broad approach using several combinations, synonyms and related search terms to "para-/intra-/testicular mesothelioma", "epididymal mesothelioma", or "para-/intra-/ testicular mesenchymal tumor/neoplasm". Non-English literature was excluded unless the abstract was available in English or the full text in French, Spanish, Italian, Portuguese, or German. Moreover, the reference lists of the identified publications were screened manually to identify additional studies. For the search strategy, see Supplementary File 1. After deduplication, two authors (JBG, JNF) screened the titles and abstracts independently and data from the same study that appeared in multiple publications were included only once. Finally, disagreements were discussed and resolved by consensus or by third-party arbitration $(\mathrm{CDF})$.

\section{Types of outcome measures included and data extraction}

Studies containing data on patient characteristics, clinicopathological features, treatment of local or metastatic disease, site of metastases, and follow-up information were eligible for this review. The data extraction sheet was developed according to the Cochrane Consumers and Communication Review Group's data extraction template. Whenever possible, data were gathered at the single-patient-level.

\section{Statistical analysis}

Categorical variables are presented as percentages and were compared using the Chi-square test of independence. The assessment of normality for continuous variables was performed visually and using the Shapiro-Wilk test. The results for normally distributed variables are expressed as mean \pm standard deviation (SD), while non-normally distributed variables are presented as median and interquartile ranges (IQRs). The independent samples $t$ test was used for normally distributed data and the Mann-Whitney $U$ test for variables with non-normally distributed data. Weighted medians were used to estimate previously processed statistical data from cohort studies as well as individual patient data from single-case reports. The optimal cut-off value for continuous variables was determined using receiveroperating curve (ROC) analyses and the maximal Youden's index (=Sensitivity + Specificity -1 ) (Youden 1950). All $p$ values $<0.05$ were considered statistically significant; all statistical tests were two-sided.

\section{Results}

\section{Included studies}

After deduplication and title as well as abstract screening, 194 of the originally identified 8,188 publications were eligible for full-text review (Fig. 1). We finally included 170 studies, resulting in a dataset of 275 patients, consisting of 140 single-case reports and 30 case series.

\section{Subgroups and location, demographics, clinical symptoms, and laboratory findings}

We identified 275 cases of MTVT, of which 227/275 $(83 \%)$ were classified as "malignant mesothelioma", with a histopathological sub-classification including epithelioid (130/227, 57\%), sarcomatoid (4/227, 2\%), and biphasic subtype (53/227, 23\%) and malignant mesothelioma of an unknown subtype (40/227, 18\%). Other histologies included well-differentiated papillary mesothelioma $(30 / 275,11 \%)$ and mesothelioma of uncertain malignant potential (13/275, $5 \%)$. Cases of benign cystic mesotheliomas $(5 / 275,2 \%)$, also known as multilocular inclusion cysts, which typically develop in the peritoneum, were also included (Table 1). The median age at diagnosis was 62 years (IQR 44-73). The 


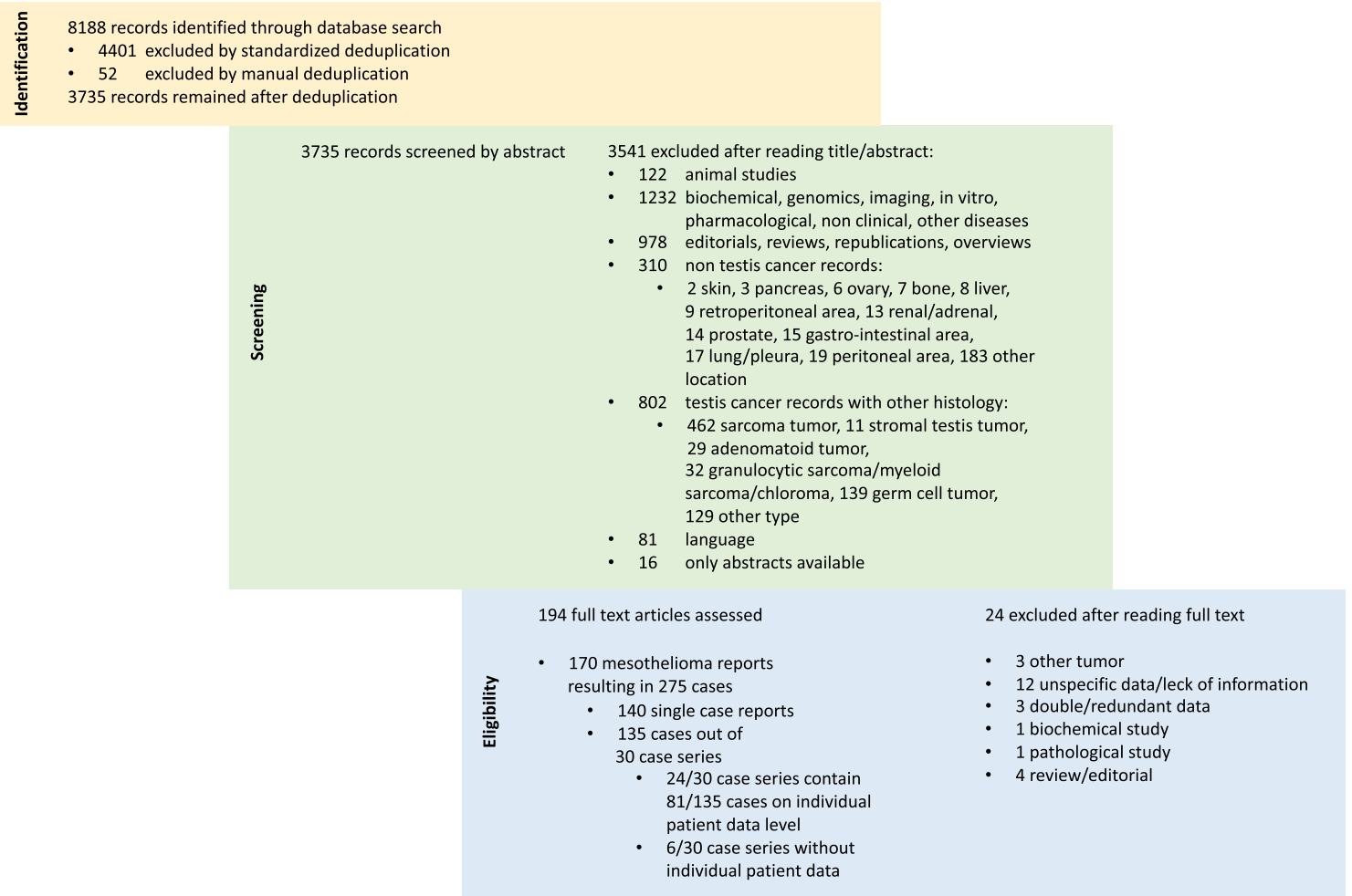

Fig. 1 Flowchart of the study selection process

tumor invaded the tunica vaginalis as the only affected structure in $170 / 275$ patients (62\%); for the remaining patients, the tunica vaginalis plus other (multiple) structures were involved: tunica albuginea and/or testis in 55/275 (20\%); epididymis in $34 / 275(12 \%)$, spermatic cord in $65 / 275$ (24\%), and the scrotum (tunica dartos and dermis) in 20/275 $(7 \%)$.

\section{Clinical presentation}

Data regarding clinical presentation were available for 259/275 patients (94\%) (Table 1). Most patients presented with a painless testicular mass/swelling, or a hydrocele (244/259, 94\%). Scrotal pain was described in 40/259 cases (15\%), and inflammation with orchitis or epididymitis in $13 / 259$ (5\%). Incidental findings during scrotal surgery represented $93 / 275$ cases (36\%). We found no reports suggesting hormonal changes leading to gynecomastia or earlier/ later puberty. Of the 154 patients with information about potential environmental risk factors of mesothelioma, 59 (38\%) described exposure to asbestos, while smoking was mentioned by 11 patients (4\%). Of the 52 patients with specific information on testicular tumor markers, lactate dehydrogenase (LDH) was elevated in $3 / 52$ cases $(6 \%)$, human chorionic gonadotropin (HCG) in $2 / 52$ cases $(4 \%)$, and alpha-fetoprotein (AFP) in $1 / 52$ cases $(2 \%)$.

\section{Local treatment and recurrence}

Most patients (170/275, 62\%) underwent radical inguinal orchiectomy as the primary local treatment, while testissparing surgery (TSS) was performed in 52/275 cases (19\%). Primary hemiscrotectomy was reported in 28/275 (10\%), transscrotal orchiectomy in two $(<1 \%)$, hydrocelectomy as the only surgical intervention in eight (3\%), and fine needle aspiration (FNA) as the diagnostic procedure without any further treatment in three (1\%). After primary TSS, secondary completion orchiectomy and secondary completion hemiscrotectomy were performed in $13(25 \%)$ and 6 patients (12\%), respectively. After primary orchiectomy, secondary hemiscrotectomy was performed in three $(6 \%)$ patients. In 12 (4\%), the primary therapy was not reported. Of the 203 patients with available follow-up data, 117 (58\%) displayed no evidence of disease after a median of 19 (IQR 9-41) months. By contrast, 67 (33\%) died after a median of 20 months (IQR 7-48). 19 men (9\%) were alive with disease after a median of 21 months (IQR 8-24). Local recurrence was observed in 35/275 patients (13\%) (Supplementary Table 1). Of the 32 patients for whom follow-up time was available, the median time from diagnosis to local recurrence was 17 months (IQR 4-24 months). The following recurrence rates were reported for the different primary treatment options: radical inguinal orchiectomy: 24/170 (14\%) after a 
Table 1 Patient characteristics overall and on individual patient-level data

\begin{tabular}{|c|c|c|}
\hline & $\begin{array}{l}\text { Individual patient level of } \\
164 \text { reports* }\end{array}$ & $\begin{array}{l}\text { Study-level data } \\
\text { from } 170 \text { reports }\end{array}$ \\
\hline Number of patients & $221(=100 \%)$ & $275(=100 \%)$ \\
\hline \multicolumn{3}{|l|}{ Histopathological (sub-)types } \\
\hline Malignant MTVT (\%) & $180 / 221(81 \%)$ & $227 / 275(83 \%)$ \\
\hline Epithelioid type (\% of malign. MTVT) & $100 / 180(56 \%)$ & $130 / 227(57 \%)$ \\
\hline Sarcomatoid type (\% of malign. MTVT) & $4 / 180(2 \%)$ & $4 / 227(2 \%)$ \\
\hline Biphasic type (\% of malign. MTVT) & $40 / 180(22 \%)$ & $53 / 227(23 \%)$ \\
\hline $\begin{array}{l}\text { Malignant MTVT of unknown subtype (\% of malign. } \\
\text { MTVT) }\end{array}$ & $36 / 180(20 \%)$ & $40 / 227(18 \%)$ \\
\hline Mesothelioma of uncertain malig. potential (\%) & $13 / 221(6 \%)$ & $13 / 275(5 \%)$ \\
\hline Well differentiated papillary MTVT (\%) & $23 / 221(10 \%)$ & $30 / 275(11 \%)$ \\
\hline Benign cystic mesothelioma (\%) & $5 / 221(2 \%)$ & $5 / 275(2 \%)$ \\
\hline Missing (\%) & $0 / 221(0 \%)$ & $0 / 221(0 \%)$ \\
\hline \multicolumn{3}{|l|}{ Age (years) } \\
\hline Median (IQR) & $62(44-73)$ & - \\
\hline Missing (\%) & $1 / 221(<1 \%)$ & \\
\hline \multicolumn{3}{|l|}{ Side } \\
\hline Left $(\%)$ & $105 / 221(48 \%)$ & $127 / 275(46 \%)$ \\
\hline Right $(\%)$ & $98 / 221(44 \%)$ & $107 / 275(39 \%)$ \\
\hline Bilateral (\%) & $5 / 221(2 \%)$ & $5 / 275(2 \%)$ \\
\hline Missing (\%) & $13 / 221(6 \%)$ & $36 / 275(13 \%)$ \\
\hline Clinical presentation & $209 / 221(95 \%)$ & $259 / 275(94 \%)$ \\
\hline Testicular enlargement/swelling/mass (\%) & $200 / 209(96 \%)$ & $244 / 259(94 \%)$ \\
\hline Scrotal pain/discomfort (\%) & $40 / 209(19 \%)$ & $40 / 259(15 \%)$ \\
\hline Epididymitis/orchitis (\%) & $13 / 209(6 \%)$ & $13 / 259(5 \%)$ \\
\hline Lymphadenopathy (\%) & $7 / 209(3 \%)$ & $7 / 259(3 \%)$ \\
\hline Incidental finding during scrotal surgery (\%) & $87 / 209(42 \%)$ & $93 / 259(36 \%)$ \\
\hline Missing (\%) & $12 / 221(5 \%)$ & $16 / 275(6 \%)$ \\
\hline \multicolumn{3}{|l|}{ Exposition } \\
\hline Asbestos (\%) & $146 / 221(66 \%)$ & $154 / 275(56 \%)$ \\
\hline Yes $(\%)$ & $51 / 146(35 \%)$ & $59 / 154(38 \%)$ \\
\hline No $(\%)$ & $95 / 146(65 \%)$ & $95 / 154(62 \%)$ \\
\hline Smoking (\%) & $11 / 221(5 \%)$ & $11 / 275(4 \%)$ \\
\hline Mean pack years (SD) & $21( \pm 13)$ & - \\
\hline Median pack years (IQR) & $23(12-29)$ & - \\
\hline Missing (\%) & $74 / 221(33 \%)$ & $121 / 275(44 \%)$ \\
\hline \multicolumn{3}{|l|}{ Tumor markers } \\
\hline AFP elevated $(\%)$ & $1 / 52(2 \%)$ & $1 / 52(2 \%)$ \\
\hline HCG elevated $(\%)$ & $2 / 52(4 \%)$ & $2 / 52(4 \%)$ \\
\hline LDH elevated $(\%)$ & $3 / 52(6 \%)$ & $3 / 52(6 \%)$ \\
\hline Missing (\%) & $168 / 221(76 \%)$ & $168 / 275(61 \%)$ \\
\hline \multicolumn{3}{|l|}{ Local primary treatment } \\
\hline Radical inguinal orchiectomy (\%) & $136 / 221(62 \%)$ & $170 / 275(62 \%)$ \\
\hline Testis-sparing surgery $(\%)$ & $41 / 221(19 \%)$ & $52 / 275(19 \%)$ \\
\hline Radical hemiscrotectomy (\%) & $26 / 221(12 \%)$ & $28 / 275(10 \%)$ \\
\hline Hydrocelectomy only (\%) & $8 / 221(4 \%)$ & $8 / 275(3 \%)$ \\
\hline Fine needle aspiration only (\%) & $3 / 221(1 \%)$ & $3 / 275(1 \%)$ \\
\hline Transscrotal orchiectomy (\%) & $2 / 221(<1 \%)$ & $2 / 275(<1 \%)$ \\
\hline Missing (\%) & $5 / 221(2 \%)$ & $12 / 275(4 \%)$ \\
\hline \multicolumn{3}{|l|}{ Local secondary treatment } \\
\hline Orchiectomy after TSS (\% of TSS) & $13 / 41(32 \%)$ & $13 / 52(25 \%)$ \\
\hline
\end{tabular}


Table 1 (continued)

\begin{tabular}{lcc}
\hline & $\begin{array}{l}\text { Individual patient level of } \\
164 \text { reports* }\end{array}$ & $\begin{array}{l}\text { Study-level data } \\
\text { from 170 reports }\end{array}$ \\
\hline Hemiscrotectomy after TSS (\% of TSS) & $6 / 41(15 \%)$ & $6 / 52(12 \%)$ \\
Hemiscrotectomy after Orchiectomy (\% of RIO) & $3 / 41(7 \%)$ & $3 / 52(6 \%)$ \\
Histopathology features & & $31 / 275(11 \%)$ \\
Necrosis (\%) & $27 / 221(12 \%)$ & $40 / 275(15 \%)$ \\
Angiolymphatic invasion (\%) & $33 / 221(15 \%)$ & $59 / 275(21 \%)$ \\
Mitotic activity (\%) & $53 / 221(24 \%)$ & $32 / 275(12 \%)$ \\
High mitotic activity (>3/10 HPF) (\%) & $26 / 221(12 \%)$ & $174 / 275(63 \%)$ \\
Missing (\%) & $143 / 221(65 \%)$ & \\
Size (mm) & & \\
Median of all histologies (IQR) & $35(15-70)$ & \\
Malignant MTVT (IQR) & $40(15-80)$ & - \\
Epithelioid type (IQR) & $38(14-100)$ & - \\
Biphasic type (IQR) & $55(27-100)$ & - \\
Well-differentiated papillary MTVT (IQR) & $15(10-29)$ & \\
Radical inguinal orchiectomy (IQR) & $38(13-78)$ & \\
Testis-sparing surgery (IQR) & $24(12-63)$ & \\
Radical hemiscrotectomy (IQR) & $40(30-150)$ & \\
Missing (\%) & $148 / 221(67 \%)$ & \\
\hline
\end{tabular}

MTVT mesothelioma of the Tunica Vaginalis Testis, RIO radical inguinal orchiectomy, TSS testis-sparing surgery, AFP alpha-fetoprotein, $H C G$ human chorionic gonadotropin, $L D H$ lactate dehydrogenase, IQR inter quartile range

*This column includes only patients of which individual patient-level data was available

median of 10 months (IQR 3-24), TSS: 6/52 (12\%) after a median of 24 months (IQR 7-30), and radical hemiscrotectomy: 3/28 (11\%) after a median of 24 months (IQR 19-55). Local recurrence in benign multicystic mesothelioma or MTVT of uncertain malignant potential was observed in one case after TSS and transscrotal orchiectomy, respectively. Salvage treatment was successful in seven patients and included local resection of recurrence and orchiectomy in five and two men with MTVT, respectively. In univariable regression analyses, only invasion of the spermatic cord or scrotum was associated with local recurrence (OR 3.21, 95\% CI 1.36-7.57, $p=0.008$ ) (Table 2).

\section{Adjuvant therapy}

Lymph-node dissection (LND) was performed in 21 men of whom 12 had inguinal LND, 8 retroperitoneal LND, 7 pelvic LND, and 6 LND in multiple locations. In 5 of the 21 patients (24\%), histological analysis showed lymph-node metastases (LNs), one without recurrence during followup. Two patients were alive with disease (one with ongoing chemotherapy and one with no further therapeutic interventions). Two patients died from metastatic spread after palliative radiotherapy and radiochemotherapy, respectively. An additional three patients without positive lymphadenectomy results displayed evidence of distant recurrence during follow-up. Thus, 6 of the 21 patients (29\%) developed systemic disease despite adjuvant LND. Local and/or metastatic relapse after adjuvant chemotherapy or chemoradiotherapy was observed in 5/9 (56\%) patients. Used chemotherapy agents included Doxorubicin, Carboplatin, or combination therapy with Adriamycin plus Cyclophosphamide or Paclitaxel, and the radiation field included the retroperitoneal and pelvic lymph nodes and pelvic region. 6/11 (55\%) men relapsed after several radiotherapy protocols including radiation of the scrotum as well as the retroperitoneal and inguinal LNs with maximum doses between 55 and $60.5 \mathrm{~Gy}$.

\section{Onset and site of metastasis}

Overall, metastatic disease was observed in $84 / 275$ patients (31\%). The following sites of metastasis were described: Retroperitoneal lymph nodes (RPLNs) (40/84, 48\%); lungs (30/84, 36\%); inguinal LNs $(23 / 84,27 \%)$; peritoneal surfaces $(18 / 84,21 \%)$; pleura $(10 / 84,12 \%)$; skin $(10 / 84,12 \%)$; liver (8/84, 10\%); pelvic LNs, mediastinal LNs and bones (each 7/84, 8\%); supraclavicular LNs (5/84, 6\%); abdominal LNs (4/84, 5\%); brain, kidneys, bladder, or pancreas (each $2 / 84,2 \%$ ), colon, rectum, spleen, and cervical LNs (each 1/84, 1\%) (Fig. 2). Regarding the subgroup of 29/84 patients (35\%) with only one metastatic site, the RPLNs (7/29, 24\%) and lungs $(7 / 29,24 \%)$ were the most common primary sites. Other solitary metastatic sites included the peritoneum (4/29, 14\%), inguinal LNs, and bones (3/29, $10 \%$ each). Two 
Table 2 Univariable regression analyses for local recurrence and metastatic disease

\begin{tabular}{|c|c|c|c|c|c|c|c|}
\hline \multirow[t]{2}{*}{ Variable } & \multirow[b]{2}{*}{$\begin{array}{l}\# \text { With available } \\
\text { variable }(\% \text { of } 221 *)\end{array}$} & \multicolumn{3}{|c|}{ Local recurrence } & \multicolumn{3}{|c|}{ Metastatic disease } \\
\hline & & OR & CI & $p$ value & OR & CI & $p$-value \\
\hline Age $(\geq 42$ years $)$ & $211(95)$ & 2.47 & $0.82-7.42$ & 0.107 & 3.02 & $1.33-6.86$ & 0.009 \\
\hline Tumor size $(\geq 49 \mathrm{~mm})$ & $67(30)$ & 3 & $0.76-11.92$ & 0.119 & 6.17 & $1.84-20.74$ & 0.003 \\
\hline $\begin{array}{l}\text { Histology } \\
\text { Malignant MTVT } \\
\text { Epithelioid type } \\
\text { Sarcomatoid type } \\
\text { Biphasic type } \\
\text { MTVT of uncertain malignant potential }\end{array}$ & $\begin{array}{l}164(74) \\
151(68) \\
97 \\
4 \\
38 \\
13(8)\end{array}$ & $\begin{array}{l}\text { Reference } \\
0.00 \\
0.82 \\
0.37\end{array}$ & $\begin{array}{l}- \\
- \\
0.30-2.26 \\
0.05-3.00\end{array}$ & $\begin{array}{l}0.814 \\
0.999 \\
0.706 \\
0.349\end{array}$ & $\begin{array}{l}\text { Reference } \\
0.00 \\
1.59 \\
0.15\end{array}$ & $\begin{array}{l}- \\
- \\
0.75-3.38 \\
0.02-1.24\end{array}$ & $\begin{array}{l}0.167 \\
0.999 \\
0.230 \\
0.079\end{array}$ \\
\hline Necrosis & $45(20)$ & 1.13 & $0.22-5.79$ & 0.880 & 8.31 & $1.58-43.62$ & 0.012 \\
\hline High mitotic index & $43(19)$ & 6.26 & $0.70-56.29$ & 0.101 & 13.36 & $1.53-116.51$ & 0.019 \\
\hline Angiolymphatic invasion & $50(23)$ & 1.26 & $0.20-7.64$ & 0.802 & 3.75 & $1.02-13.80$ & 0.047 \\
\hline $\begin{array}{l}\text { Location/involvement } \\
\text { Tunica vaginalis only } \\
\text { Invasion of tunica albuginea } \\
\text { and/or testis } \\
\text { Invasion of epididymis } \\
\text { Invasion of spermatic cord or scrotum }\end{array}$ & $\begin{array}{l}211(95) \\
121(55) \\
23(10) \\
19(9) \\
48(22)\end{array}$ & $\begin{array}{l}\text { Reference } \\
2.47 \\
0.99 \\
3.21\end{array}$ & $\begin{array}{l}- \\
0.78-7.80 \\
0.20-4.76 \\
1.36-7.57\end{array}$ & $\begin{array}{l}0.044 \\
0.124 \\
0.986 \\
0.008\end{array}$ & $\begin{array}{l}\text { Reference } \\
0.61 \\
0.78 \\
1.56\end{array}$ & $\begin{array}{l}- \\
0.21-1.76 \\
0.26-2.32 \\
0.78-3.11\end{array}$ & $\begin{array}{l}0.335 \\
0.357 \\
0.655 \\
0.207\end{array}$ \\
\hline \multicolumn{8}{|l|}{ Local recurrence } \\
\hline Recurrence versus no recurrence & 209 (95) & - & - & - & 4.35 & $2.00-9.44$ & $<0.001$ \\
\hline
\end{tabular}

OR odds ratio, $C I$ confidence interval, MTVT mesothelioma of the tunica vaginalis testis, TSS testis-sparing surgery

*Cases with individual patient-level data

case reports each $(2 / 29,7 \%)$ described singular metastasis of MTVTs to the brain, the liver, and the mediastinal LNs. The exact timing of metastatic disease in relation to diagnosis was described in 52/84 (62\%) patients. Metastatic disease at initial diagnosis was described in $18 / 52$ men (35\%), whereas $34 / 52$ men $(65 \%)$ presented with metastatic recurrence during a median follow-up of 17 months (IQR 10-29).

\section{Risk factors for metastatic disease}

Patients with well-differentiated papillary mesothelioma or benign multicystic mesothelioma did not develop metastatic disease. Regarding the different histologic sub-classifications of malignant MTVT on individual patient-level data, 34/100 patients (34\%) with epithelioid type and 18/40 (45\%) with biphasic type exhibited metastatic behaviour. The remaining patients with metastatic disease were either unclassified or belonged to other rare subtypes of malignant MTVT [15/36 (42\%) malignant MTVT without subtype information, 1/13 (8\%) MTVT of uncertain malignant potential].

Individual patient-level data to analyse further risk factors were available for 68 men with metastatic MTVT. Risk factors for metastasis included age $\geq 42$ years (OR 3.02, 95\% CI 1.33-6.86, $p=0.009)$, tumor size $\geq 49 \mathrm{~mm}(\mathrm{OR} 6.17,95 \% \mathrm{CI}$ 1.84-20.74, $p=0.003$ ), and presence of necrosis (OR 8.31, 95\% CI 1.58-43.62, $p=0.012$ ), high mitotic index (OR
$13.36,95 \%$ CI $1.53-116.51, p=0.019)$, or angiolymphatic invasion (OR 3.75, 95\% CI 1.02-13.80, $p=0.047$ ), and local recurrence (OR 4.35, 95\% CI 2.00-9.44, $p<0.001$ ).

\section{Treatment and outcome in men with metastatic disease}

During a median follow-up of 18 months (IQR 7-43), 55/84 metastatic MTVT patients (65\%) died, and 13/84 $(15 \%)$ were alive with disease, while 11 patients were either lost to follow-up or without information on survival. Complete remission in the metastatic setting was reported in five men (Supplementary Table 2): the first patient (\#7) with an epithelial MTVT was treated with an orchiectomy and RPLND showing involvement of one retroperitoneal lymph node. Data about staging CT at diagnosis as well as information about further treatment during follow-up were not available. This patient remained free of disease for at least 66 months (Bertolotto et al. 2016). The second patient (\#12) with an epithelioid MTVT developed mediastinal LN metastases 36 months after primary radical orchidectomy. After six cycles of Cisplatin and Pemetrexed and maintenance with Pemetrexed, there was no evidence of disease after 42 months of follow-up (Doris et al. 2015). The third patient (\#35), with a malignant MTVT of unknown histological subtype and peritoneal metastases at initial staging, was treated with testis-sparing surgery, peritonectomy, and 


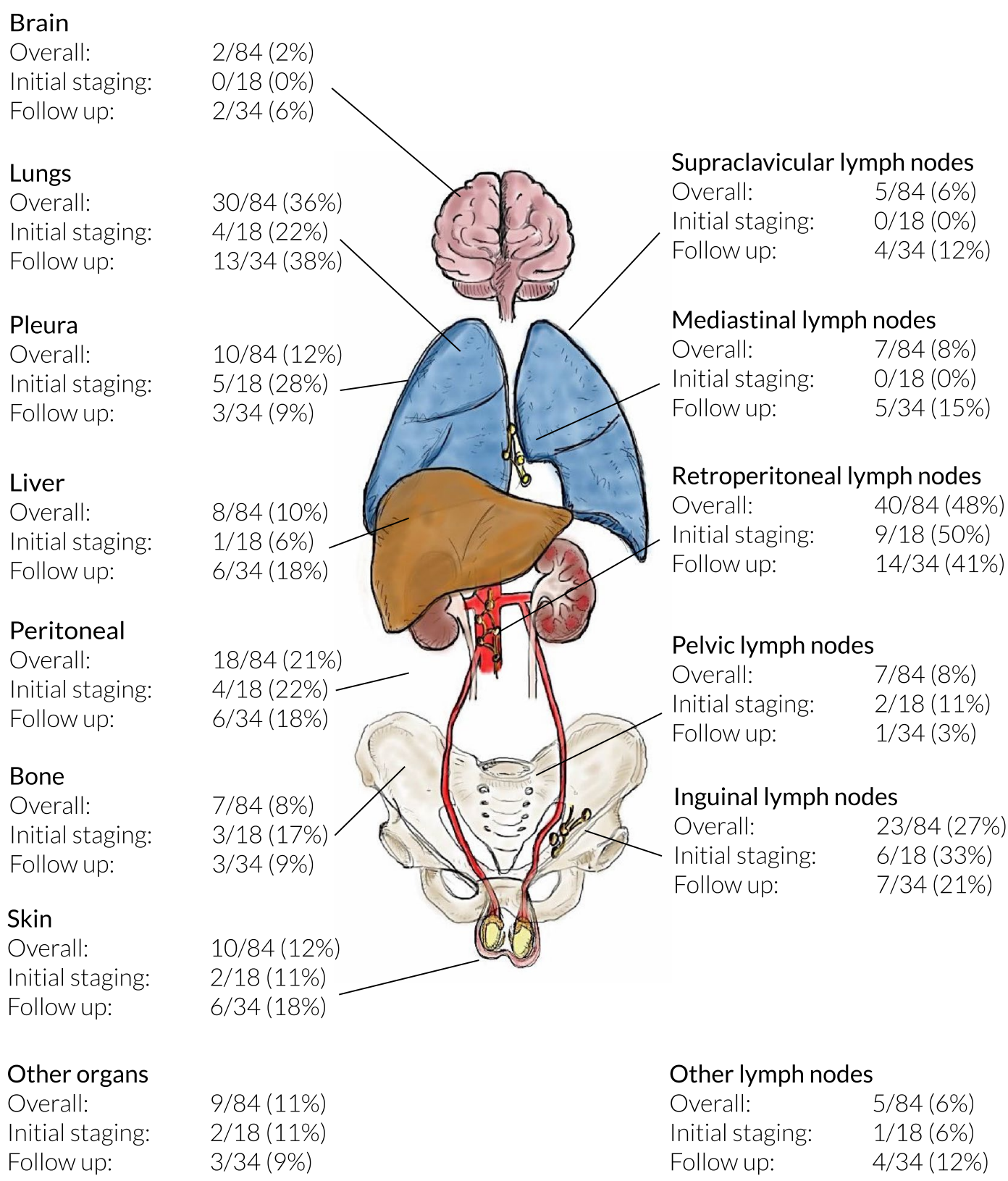

Fig. 2 Anatomical locations of metastatic sites at initial staging and during follow-up. Affected organs are listed on the left and lymph-node locations on the right

intraperitoneal hyperthermic perfusion chemotherapy. He remained free of disease after a follow-up of 18 months (Schure et al. 2006). The fourth patient (\#42), who had epithelioid MTVT with extension to the spermatic cord in the inguinal canal and metastatic spread within the pelvis and the abdomen, was initially treated with hemiscrotectomy with resection of the inguinal mass and abdominal cytoreductive surgery with extensive stripping all peritoneal surfaces followed by intra-operative, intra-peritoneal chemotherapy using Cisplatin and Doxorubicin. This patient was recurrence-free survival at 5 years (Sebbag et al. 2001).
The fifth patient (\#60), with epithelioid MTVT invading the tunica albuginea, the spermatic cord and external scrotal layers, was treated with a primary hemiscrotectomy. After resection of visible metastases in the retroperitoneum, six cycles of chemotherapy with Adriamycin and Cyclophosphamide and radiation therapy of 25 Gy over 3 weeks, the patient was disease-free at 36 months (Lopez et al. 1995). Of metastatic cases with available follow-up, 20 out of 21 (95\%) patients treated with surgery only and 64 out of 68 (94\%) patients treated with multimodal therapies progressed. 


\section{Discussion}

Our analysis of published case series is the largest and most comprehensive summary of the available literature regarding MTVT. It also extends the previous reviews (Bisceglia et al. 2010; Plas et al. 1998b; Vimercati et al. 2019; Zhang and Goldsztajn 2019), and therefore, several important insights can be discussed.

The highest incidence was found in men in their sixties; however, there is a wide age range, even patients under 20 years can be affected. MTVT usually presents as a testicular mass or is incidentally discovered during or after inguino-scrotal surgery. Therefore, the primary local treatment depends on the clinical scenario, but treatment should also be planned in the knowledge that MTVT can show multifocal growth and that local recurrence might be associated with metastatic disease.

In men with first presentation, several recommendations might help to guide clinicians in decision-making, but those are based on very limited evidence or expert opinion only. Men in whom MTVT is suspected at initial presentation, primary hemiscrotectomy, and en-bloc orchidectomy should be performed. In men with suspicious findings discovered during surgery, we recommend completing the hydrocelectomy as planned and only proceeding thereafter to a hemiscrotectomy if the final histopathology confirms the diagnosis of MTVT. This is mainly since most men scheduled for routine scrotal surgery such as hydrocelectomy are not consented for a hemiscrotectomy and the diagnostic accuracy of intraoperative frozen section remains ill-defined. For men with an incidental diagnosis of MTVT in the hydrocelectomy specimen, we recommend timely completion of a hemiscrotectomy because of potential tumor seeding.

Well-differentiated papillary mesothelioma and benign multicystic mesothelioma are clearly benign entities that require complete resection as definitive therapy; thereafter, only follow-up for local relapse is advisable. By contrast, malignant MTVT exhibited metastatic spread in one-third of cases, suggesting repeated cross-sectional imaging of the chest and abdomen is required for staging and follow-up. The available data do not support the regular use of adjuvant treatment. Half of all cases had involved retroperitoneal LNDs, and only in a proportion, other organs were also affected. Despite the use of RPLND, all patients treated with lymphadenectomy, who did not receive additional chemoor radiotherapy, progressed. Thus, RPLND as monotherapy does not have the potential to cure patients. Only a few men received adjuvant chemo- or radiotherapy which does not allow any conclusions.

Patients with metastatic disease have a poor prognosis, and standard treatment recommendations are not available. Given the experience in pleural mesothelioma and the scarce data on MTVT, surgical resection within a multimodal treatment approach can be offered; however, the choice of chemotherapy and the role of radiotherapy remain unclear. Pleural mesothelioma guidelines recommend Platinum, Pemetrexed plus minus Bevacizumab (Opitz et al. 2020) and in one case Pemetrexed led to complete response. Additionally, Cisplatin and Doxorubicin or Adriamycin and Cyclophosphamide could be suggested as a few responses have been observed.

\section{Limitations}

The published literature only consists of retrospective case reports and small case series with a low number of outcome events and missing single-patient data, and multivariable analysis was therefore not possible. Our search strategy was designed and reviewed by both clinicians and librarians, and was predefined in a peer-reviewed protocol. However, the possibility remains that not all potentially relevant studies were identified; this is an additional source of potential bias. Larger and more consistent datasets are needed to develop prediction models involving several risk factors. Nevertheless, the current analysis provides a unique overview of the published experience with MTVTs. Due to the absence of prospective trials, we recently opened the OrphAn Testis Histologies (OATH) to provide more conclusive recommendations regarding clinical course, management, and follow-up of these rare entities. We encourage collaborators to contribute data regarding patients with rare testis cancer histologies (http://bit.ly/OATH-registry).

Supplementary Information The online version contains supplementary material available at https://doi.org/10.1007/s00432-021-03533-6.

Acknowledgements We would like to thank the clinical medical librarian S. Klein for her expertise and support during both planning and execution of our systematic literature search.

Author contributions CDF, TH, and JBG contributed to the study conception and design. Material preparation, data collection, and analysis were performed by JNF, JBG, and CDF. The same authors drafted and wrote the manuscript after critical revision of all authors. PKB, $\mathrm{PO}, \mathrm{AL}$, and JB provided specialists' opinion and assistance on histopathology and clinical oncology. All authors read and approved the final manuscript.

Funding Open Access funding provided by Universität Zürich. Furthermore, the authors did not receive any other support from any organization for the submitted work.

Data availability The datasets used and/or analysed during the current study are available from the corresponding author on reasonable request. 


\section{Compliance with ethical standards}

Conflicts of interests The authors have no relevant financial or nonfinancial interests to disclose. The results presented in this paper have not been published previously.

Ethics approval According to the Swiss Ethics Committee and the Ethics Committee of Zurich (KEK), this study does not require a formal ethics approval. The study was performed in accordance with the Declaration of Helsinki.

Open Access This article is licensed under a Creative Commons Attribution 4.0 International License, which permits use, sharing, adaptation, distribution and reproduction in any medium or format, as long as you give appropriate credit to the original author(s) and the source, provide a link to the Creative Commons licence, and indicate if changes were made. The images or other third party material in this article are included in the article's Creative Commons licence, unless indicated otherwise in a credit line to the material. If material is not included in the article's Creative Commons licence and your intended use is not permitted by statutory regulation or exceeds the permitted use, you will need to obtain permission directly from the copyright holder. To view a copy of this licence, visit http://creativecommons.org/licenses/by/4.0/.

\section{References}

Bertolotto M et al (2016) Imaging of mesothelioma of tunica vaginalis testis. Eur Radiol 26:631-638

Bisceglia M, Dor DB, Carosi I, Vairo M, Pasquinelli G (2010) Paratesticular mesothelioma. Report Case Comprehens Rev Lit Advanc Anatom Pathol 17:53-70

Doris M, Antonogiannaki EM, Katsenos S (2015) Paraneoplastic nephritic syndrome and concurrent solitary mediastinal lymph node metastasis from mesothelioma of testis diagnosed by endobronchial ultrasound: unusual manifestations of an extremely rare tumor. J Cancer Res Therap 11:670

Gurdal M, Erol A (2001) Malignant mesothelioma of tunica vaginalis testis associated with long-lasting hydrocele: could hydrocele be an etiological factor? Int Urol Nephrol 32:687-689

Lopez JI, Angulo JC, Ibanez T (1995) Combined therapy in a case of malignant mesothelioma of the tunica vaginalis testis. Scand $\mathbf{J}$ Urol Nephrol 29:361-364
Moher D et al. (2015) Preferred reporting items for systematic review and meta-analysis protocols (PRISMA-P) 2015 statement systematic reviews $4: 1$

Moher D, Liberati A, Tetzlaff J, Altman DG (2009) Preferred reporting items for systematic reviews and meta-analyses: the PRISMA statement. J Clin Epidemiol 62:1006-1012. https://doi. org/10.1016/j.jclinepi.2009.06.005

Opitz I et al (2020) ERS/ESTS/EACTS/ESTRO guidelines for the management of malignant pleural mesothelioma. Eur J Cardiothorac Surg 58:1-24. https://doi.org/10.1093/ejcts/ezaa158

Plas E, Riedl CR, Pfluger H (1998a) Malignant mesothelioma of the tunica vaginalis testis: review of the literature and assessment of prognostic parameters. Cancer 83:2437-2446

Plas E, Riedl CR, Pflüger H (1998b) Malignant mesothelioma of the tunica vaginalis testis: review of the literature and assessment of prognostic parameters. Cancer Interdisc Intern J Am Cancer Soc 83:2437-2446

Schure PJ, van Dalen KC, Ruitenberg HM, van Dalen T (2006) Mesothelioma of the tunica vaginalis testis: a rare malignancy mimicking more common inguino-scrotal masses. J Surg Oncol 94:162-164

Sebbag G, Yan H, Shmookler BM, Sugarbaker PH (2001) Malignant mesothelioma of the male genital tract: report of two cases. Urol Oncol 6:261-264. https://doi.org/10.1016/S1078-1439(01)00123 $-5$

Vimercati L, Cavone D, Delfino MC, De Maria L, Caputi A, Ferri GM, Serio G (2019) Asbestos exposure and malignant mesothelioma of the tunica vaginalis testis: a systematic review and the experience of the Apulia (southern Italy) mesothelioma register Environmental Health 18:78

Youden WJ (1950) Index for rating diagnostic tests. Cancer 3:32-35. https://doi.org/10.1002/1097-0142(1950)3:1\%3c32::aid-cncr2 $820030106 \% 3 \mathrm{e} 3.0 . c 0 ; 2-3$

Zhang C, Goldsztajn HJ (2019) Study of the clinical characteristics of scrotal mesothelioma J Clin Oncol 37

Publisher's Note Springer Nature remains neutral with regard to jurisdictional claims in published maps and institutional affiliations. 